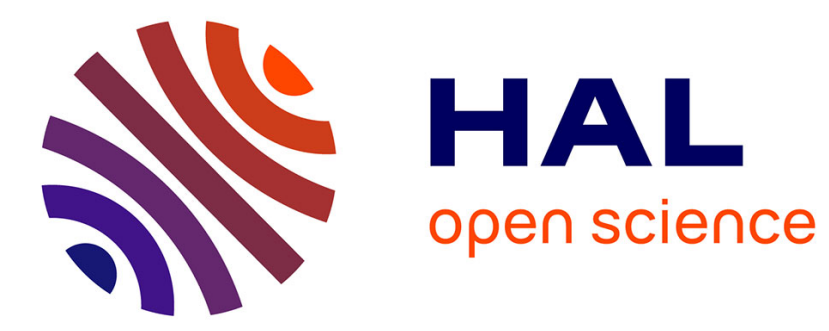

\title{
L'autochtonie, la cité et les origines humaines : d'un discours politique à un discours cosmologique
}

\author{
Karin Mackowiak
}

\section{To cite this version:}

Karin Mackowiak. L'autochtonie, la cité et les origines humaines : d'un discours politique à un discours cosmologique. Mètis - Anthropologie des mondes grecs anciens, 2016, N.S.14, pp.135-157. 10.4000/books.editionsehess.4212 . halshs-02111793

\section{HAL Id: halshs-02111793 \\ https://shs.hal.science/halshs-02111793}

Submitted on 1 Dec 2021

HAL is a multi-disciplinary open access archive for the deposit and dissemination of scientific research documents, whether they are published or not. The documents may come from teaching and research institutions in France or abroad, or from public or private research centers.
L'archive ouverte pluridisciplinaire HAL, est destinée au dépôt et à la diffusion de documents scientifiques de niveau recherche, publiés ou non, émanant des établissements d'enseignement et de recherche français ou étrangers, des laboratoires publics ou privés. 


\section{L'autochtonie, la cité et les origines humaines : d'un discours politique à un discours cosmologique}

Karin Mackowiak

Université de Franche-Comté, ISTA - EA 4011

: autochtonie, présocratique, cité, anthropogonie, cosmologie

: autochtony, presocratic, city, cosmology

Les récits d'autochtonie de Grèce ancienne ont fait l'objet d'études exhaustives sur le plan politique mais guère sur celui cosmologique qui éclairerait pourtant les représentations des origines humaines sous un autre angle. L'historien des religions et de la société doit s'en emparer pour en évaluer l'originalité. Croisée à l'univers présocratique, l'autochtonie évolue dans une vision spécifique du monde à l'époque archaïque, qui révèle la dimension cosmologique de la cité et de la créature humaine en Grèce ancienne.

Ancient Greek myths telling autochtony were hardly considered outside the political point of view although another understanding of such representations is possible - cosmologically seen. The historian of religions taking over from social studies can take hold of this subject which motivates reflections concerning its original feature. Compared with the presocratic's cosmology, autochtony myths belong to an archaic world view in which the idea of polis acquire a fully cosmological sense and that of mankind another consistency.

\section{Remarques historiographiques et méthodologiques}

En quoi l'autochtonie constitue-t-elle une anthropogonie ?

Comment les récits des origines de Thèbes en particulier peuvent-ils apporter une réponse à cette question?

Ces questions présentent plusieurs intérêts, d'ordre historiographique d'abord : jusque-là, l'autochtonie a pour l'essentiel fait l'objet d'études historiennes focalisées sur sa dimension politique $^{1}$. Mais cet imaginaire n'a-t-il pas encore une autre dimension, d'ordre cosmologique ? Un tel angle d'approche, qui sollicite davantage l'histoire des religions, a l'avantage de renouveler l'historiographie sur un point précis: un certain nombre d'études ont déjà comparé l'autochtonie grecque aux anthropogonies orientales et ont déduit le peu d'importance apportée par les Hellènes aux origines de la créature humaine au motif qu'il n'y a pas trace de récits grecs

\footnotetext{
${ }^{1}$ Récemment, voir par exemple : Sophie GotTELAND, "L'origine des cités grecques dans les discours athéniens ", in Valérie Fromentin, Sophie Gotteland (éd.), Origines gentium, Paris, 2001, p. 79-93 ; Sonia Darthou, «Retour à la terre : fin de la Geste d'Érechthée », Kernos 18, 2005, p. 69-83 ; Violaine Sebillotte Cuchet, Libérez la patrie! Patriotisme et politique en Grèce ancienne, Paris, 2006, chapitre IX. Sur la nécessité de désenclaver l'autochtonie de sa dimension politique, voir Claudine LEDUC, «Poésie et politique dans le mythe de l'autochtonie athénienne ", in Lydie Bodiou, Véronique MeHL (dir.), La religion des femmes en Grèce ancienne : mythes, cultes et société, Rennes, 2009, p. 161-170.
} 
parlant de l'apparition du premier homme, sur un plan général2 ${ }^{2}$ Or, cette supposée lacune constatée pour l'essentiel dans le cadre de la culture grecque archaïque n'est pas convaincante.

À l'historien anthropologue qui reprend ce point, il n'échappe pas que les questions furent mal posées en raison, notamment, de schémas culturels hérités du judéo-christianisme. Pourquoi ces derniers seraient-ils opératoires en Grèce ancienne ? Ce décalage a nourri la vision, erronée, de Grecs "mauvais peintres", négligents dans l'esquisse d'un "Adam” sur le grand tableau de la création du monde. Or, une conception (fut-elle différente) de la création du monde ou un système de représentations élaboré qui explique l'apparition de l'humanité aux origines existe bien chez les Grecs $^{3}$ : si les récits des origines de l'homme n'apparaissent certes pas dans le cadre d'une version canonique mais, au contraire, de façon dispersée sur les plans historique comme géographique, ces traditions sont néanmoins révélatrices de la présence de la question de la création de l'être humain chez les Grecs, ne serait-ce que par le biais du récit de la première femme, Pandora, attesté chez Hésiode (Théogonie 570-593 ; Les Travaux et les Jours 90-105). Reste que la présente étude se borne à traiter de la créature mâle puisque les réflexions comparatistes entre imaginaires grec et oriental sont parties de là. Je souhaiterais donc montrer, ici, que l'imaginaire du premier homme est simplement construit, chez les Grecs, d'une manière différente de celle à laquelle le monothéisme nous a habitués. C'est d'abord, comme on le sait, dans le cadre de récits de fondations politiques comme le montre en particulier l'imaginaire d'autochtonie. Mais il reste à savoir, ensuite, comment l'autochtonie formule en tant quanthropogonie en soi un discours cosmologique, et comment elle le fait au sein même et audelà du discours politique dans lequel elle évolue.

Outre le décalage d'un mode de pensée spécifiquement grec, ces questions relèvent encore d'un autre intérêt qui est d'ordre méthodologique : si des recours ponctuels à certaines croyances orientales peuvent, dans la présente étude, nous aider à cerner les spécificités grecques d'une vision du monde, ce genre de comparaison ne suffit pas à mettre en valeur la construction originale de l'imaginaire grec ni son mode de constitution historique depuis l'époque archaïque. C'est pourquoi il est intéressant de se référer aussi à une autre comparaison qui s'appuie sur les théories des origines du monde et de l'homme des Présocratiques. Or, scruter cet imaginaire-là a plusieurs avantages : il nous permet de remonter aux sources archaïques de l'imaginaire grec d'anthropogonie et d'évaluer la proximité de représentations mythologiques d'un côté et d'idées cosmologiques réputées savantes de l'autre; cette comparaison nous permet également de revenir sur un imaginaire d'autochtonie authentiquement archaïque, celui de Thèbes en Béotie

\footnotetext{
${ }^{2}$ Depuis Peter Walcot, Hesiod and the Near East, Cardiff, 1966, p. 62 sq. ou Geoffrey S. KIRK, The Nature of Greek Myths, Harmondsworth, 19805, p. 140 sq. et p. 273 jusqu'à Bernard DEFORGE, Le commencement est un dieu. Un itinéraire mythologique, Paris, 1990, p. 156 repris par Patrick Kaplanian, Mythes grecs d'origine I. Prométhée et Pandore, Paris, 2011, p. 242254. Voir également les points de vue d'Alain Moreau, Mythes grecs I. Origines, Montpellier, 1999, p. 112 sq. ou de Michael P. Schmude, «Der Mensch-Mängelwesen oder Krone der Schöpfung », Anregung, Zeitschrift für Gymnasialpädagogik 43, 1997, p. 91-94.

${ }_{3}$ À des échelles et époques variées : voir la création des hommes par Prométhée (Apollodore, Bibliothèque I, 7, 1 ; Ovide, Métamorphoses I, 78) ou par les dieux (Platon, Protagoras 320d) ou encore à partir des cendres des Titans (Hymne homérique à Apollon 335 sq). Il faut aussi songer au mythe des races d'Hésiode (Travaux et Jours 106-201) dont la signification peut cependant être plus compliquée qu'une simple anthropogonie : voir Alain BALLABRIGA, "La fabrication de l'humain dans les mythes orientaux et grecs », Études de Lettres 3-4, 1998, p. 3347 ; du reste, je m'attèle à cette question ailleurs, dans un travail en cours de réalisation.
} 
où le récit des Spartes thébains est déjà formé depuis le $\mathrm{VII}^{\mathrm{e}}-\mathrm{VI}^{\mathrm{e}}$ s. av. J.-C. au moins ${ }^{4}$; sans oublier que telle comparaison n'a pour l'instant jamais été tentée. Or, cette méthode nous permet de dépasser la dimension politique de l'autochtonie. Les Présocratiques nous permettent de comprendre, dans le cadre d'une synthèse même modeste, quelle était l'originalité des Grecs en matière de cosmologie et d'anthropogonie puisque se dégage l'idée selon laquelle les premiers hommes sont nés du sol comme des plantes. Enfin, en dépit des problèmes d'accès qu'ils posent, les Présocratiques, reconnus aujourd'hui comme exprimant une forme de pensée traditionnelle en son originalité ${ }^{5}$, permettent d'éclaircir certains héritages archaïques qui subsistent dans des sources mythographiques tardives mais incontournables.

Il faut finir cette introduction sur-ce : étudier l'autochtonie thébaine archaïque ne dispense pas de prendre en compte des textes d'époques classique, hellénistique et romaine ${ }^{6}$. Les sources proprement thébaines sont rares $^{7}$, et les récits plus tardifs présentent des évolutions par exemple politiques - on pense surtout au point de vue athénien qui n'a pas manqué de donner sa version de l'autochtonie thébaine. Mais au fil des siècles qui ont constitué cette autochtonie, les élaborations poétiques de la mythologie doivent aussi nous intéresser du point de vue de leurs permanences culturelles, dans leur faculté à avoir conservé des représentations archaïques où s'imposait la vision d'“hommes-plantes" nés du sol. Selon cet angle de vue, les sources mythologiques, comme les autres, continuent à explorer un discours implicite sur la place de l'homme dans le cosmos. Un dernier aspect méthodologique de la présente étude se trouve bien là : il faut expliciter cette dimension anthropogonique, l'extraire véritablement du substrat de mythographies qui ont amoindri la dimension cosmologique de l'autochtonie selon leurs logiques propres sans toutefois jamais l'oublier.

\footnotetext{
${ }^{4} \mathrm{La}$ plus ancienne source est Stésichore d'Himère (fr. 195 Page : VII ${ }^{\mathrm{e}}$ s. av. J.-C) suivie de Phérécyde d'Athènes (FGrHist $3 \mathrm{~F} 22 \mathrm{a}$ et $\mathrm{c}: \mathrm{VI}^{\mathrm{e}}$ s. av. J.-C) et d'Hellanicos de Lesbos (FGrHist $4 \mathrm{~F} 51$ : $\mathrm{V}^{\mathrm{e}}$ s. av. J.-C)... après qui viennent les Tragiques (infra n. 7).

${ }^{5}$ Réfutant notamment les dimensions anthropomorphique et généalogique du divin : voir les mises au point de Keimpe Algra, "The Beginnings of cosmology », in Keimpe Algra, Antony A. Long (ed.), The Cambridge Companion to Early Greek Philosophy, Cambridge, 1999, p. 45-65 et de Glenn W. Most, "The poetics of early Greek philosophy ", in ibid., p. 332-362. Sur la difficulté d'accéder parfois à la pensée présocratique, voir le cas d'Héraclite selon Serge N. Mouraviev, "Comment interpréter Héraclite: vers une méthodologie scientifique des études héraclitéennes ", in Konstantine J. Boudouris (ed.), Ionian Philosophy, Athènes, 1989, p. 270-279.

6 Pour l'époque classique, surtout : Eschyle, Les Sept contre Thèbes 412-414 et Euripide, Phéniciennes 5-6 ; 203-205; 638-689; 818-832; 930-941; 1006-1008; 1062-1065 et Bacchantes 170-172; 263-265; $540 ; 1025-1026$; 1274. Pour les périodes hellénistique et romaine, les récits les plus complets sont: Apollodore, Bibliothèque III, 4, 1-2 ; Ovide, Métamorphoses III, 6-136 ; Pausanias, Périégèse IX, 5, 1-3 et 12, 1-3 ou Nonnos, Dionysiaques II, 663-668; IV, 349-463. Les scholies consacrées aux tragédies athéniennes sont également précieuses (infra n. 9, 11, 14 ou 18).

${ }^{7}$ De Thèbes, nous est guère parvenu que Pindare, Isthmiques I, 30 ; VII, 14 et Hymnes, I (= fr. 29, 2 Snell).
} 


\section{De l'univers mythique aux Présocratiques : réflexions sur une approche similaire de l'homme et du cosmos}

Parler d'hommes-plante est justifié par la mythographie qui nous parle des Spartes : ces hommes originels issus du sol de Thèbes sont nés de dents de serpent semées sur la terre ${ }^{8}$. L'emploi des verbes pour décrire ce geste varie dans les textes mais un certain nombre de récits utilise $\sigma \pi \varepsilon \varepsilon^{\prime} \varrho \omega^{9}$ comme pour un acte d'ensemencement : associées à une fécondation, les dents du serpent d'Arès ont été vues par certains mythographes comme des graines éparpillées sur une matrice, la terre, dont l'aspect maternel lui donne la faculté de générer des autochtones ${ }^{10}$. À $\sigma \pi \varepsilon i ́(\omega$, les sources ajoutent la pousse aussitôt active des Spartes : ceux-ci croissent littéralement du sol ( $\dot{\alpha} v \alpha \phi u ́ o v \sigma \iota v)$ suivant une représentation qui fait converger production végétale et " gestation» dans un ventre origine ${ }^{11}$.

Une première ligne de structure cosmologique se dégage de ces représentations : la croyance archaïque en un cosmos vivant. Or, les Présocratiques avaient élaboré une vision du monde similaire : pour Thalès c'est l'eau qui est à l'origine de toute chose, pour Anaximène, l'air tandis que pour Anaximandre il s'agit de l'infini : ces théories ioniennes considéraient le monde comme capable d'engendrer en fonction d'un principe spermique susceptible de faire naitre les premiers êtres vivants ${ }^{12}$. Ce genre de vision habite encore la pensée de Lucrèce qui, au $\mathrm{I}^{\text {er }} \mathrm{s}$. av. J.-C., continue à mettre en parallèle la nova tellus qui fait pousser les herbes (De rerum natura $\mathrm{V}, 811-815)$ et l'image de la terre/mère ou de la terre/femme qui enfante et nourrit les hommes : après être venus au jour, les premiers êtres léchaient en effet la terre, celle-ci leur procurant un sucum consimilem lactis (808-809). Imprégné d'épicurisme et de l'enseignement de Démocrite, Lucrèce fait la synthèse entre poésie et connaissances scientifiques : il donne à la nature des contours philosophiques assez peu différents des représentations mythologiques ${ }^{13}$, à la manière des Présocratiques.

${ }^{8}$ Des sources athéniennes aux sources thébaines, l'histoire des Spartes ne varie guère : les autochtones sont nés de la terre et des dents du serpent d'Arès, gardien d'une source, tué par le héros Cadmos. La naissance des Spartes est suivie du mariage de Cadmos et d'Harmonie déjà connu de Pindare, Pythique III, 91-92.

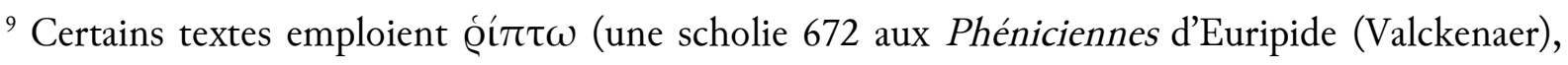

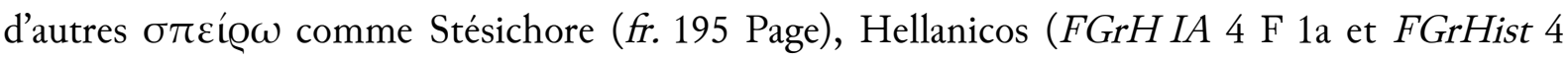
F 51), Phérécyde (FGrHist 3 F 22a), scholie 1206 à l'Alexandra de Lycophron (Scheer). Une étude spécifique est prévue sur l'usage du premier verbe et la façon dont il peut être compris.

${ }^{10}$ Sur l'aspect maternel de la terre dont les textes grecs rendent compte tantôt sur le plan biologique (descendance/filiation) tantôt sur celui productif (karpophoros), voir respectivement Pierre Chantraine, "Les noms du mari et de la femme, du père et de la mère en grec », REG 59-60, 1946-1947, p. 219-250 ; Sebillotte Cuchet (supra, n. 1), p. 270-276 ; Stella GEorgoudi, "Gaia/Gê. Entre mythe, culte et idéologie », in Synnøve Des Bouvrie (ed.), Myth and Symbol. Symbolic phenomena in ancient Greek culture. 1, Bergen, 2002, p. 113-134.

${ }^{11}$ Phérécyde (FGrHist 3 F22a et FGrHist 3 F22c), Hellanicos (FGrHist 4 F1b : ع̌ $\phi v \sigma \alpha v$ ) ou la scholie 5 aux Phéniciennes d'Euripide (Valckenaer). Pour la gestation, voir Eschyle (fr. 731b

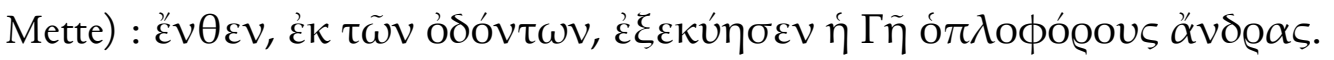

12 Diels Kranz 12 A9 pour la théorie d'Anaximandre. Pour Anaximène: DK 13 B2. Pour Thalès : DK 11 A12. 
Aussi n'est-il pas étonnant de trouver une seconde ligne de structure cosmologique commune entre le mythe d'autochtonie et les Présocratiques: la représentation suivant laquelle la naissance des premières créatures s'amalgame à une différenciation des éléments et évolue de l'informe vers la forme.

Il est alors intéressant d'observer les données de l'autochtonie thébaine : le lieu de la naissance des Spartes était situé près d'une source, soit : en un lieu humide où l'eau était peu distincte de la terre. Cette source était gardée par un serpent monstrueux dont les dents ont été répandues sur une glèbe molle. Le fragment 195 (Page) de Stésichore donne une place à Athéna dans la naissance des Spartes. Ce détail est précieux car il renvoie sans doute à l'existence d'un sanctuaire d'Athéna près des portes thébaines, là où la source se trouvait dès l'antiquité ${ }^{14}$, dans une zone de terrain boueux. L'autochtonie thébaine semble donc bien participer à la vision selon laquelle les premiers hommes sont nés d'une différenciation d'éléments terreux et aqueux, une émergence de la glèbe dont ils se sont progressivement extraits. Or, ce type d'anthropogonie est attesté ailleurs en Béotie, chez Hésiode notamment ${ }^{15}$.

Mais c'est sans doute le livre III, 1391-1398 des Argonautiques d'Apollonios de Rhodes rapportant la naissance des Colques, jumeaux poétiques des Spartes, qui est le plus clair à ce sujet. Si des innovations poétiques, dues à une esthétique toute alexandrine, influencent ce livre, elles ne semblent pas affecter les figures guerrières des Colques marquées par un héroïsme épique à la manière archaïque thébaine ${ }^{16}$. La description de leur corps émergeant de la masse terrienne est éloquente: les Colques ont d'abord un corps mou et flasque; mis à part le vocabulaire médical hellénistique qui concerne la description de leur tête, les pieds de ces autochtones font en revanche valoir la progressive différenciation de leur corps d'avec la glèbe d'où ils sont issus : dans ce passage agit une sorte de métaphore poétique de la dessiccation ${ }^{17}$.

Sur ce point, Apollonios de Rhodes semble donc être resté fidèle à une vision archaïque où la matrice originelle, génératrice, est aussi synonyme d'obscur, de mélangé, d'informe. Le serpent d'Arès lui-même y fait songer si l'on se rappelle une figure qui lui est proche, Python, pourrissant sur le sol delphique sans plus désormais s'en distinguer après la victoire d'Apollon. L'Hymne homérique 363-374 expose une association d'idées entre le serpent et le sol dans lequel il vit : c'est une sorte de confusion encore redoublée par les formes onduleuses et fuyantes de l'animal en tous points opposées à la netteté des contours ${ }^{18}$. Le serpent est par définition ce

${ }^{13}$ Marie-José BÉNÉJAM-Bontems, "Les origines de l'homme selon Lucrèce », in Jean-Michel Galy, Antoine Thivel (éd.), Les origines de l'homme d'après les anciens, Nice, 1998, p. 147152.

${ }^{14}$ Avec confirmation de la scholie 1068 aux Phéniciennes d'Euripide = scholie 487a aux Sept contre Thèbes d'Eschyle (Langwitz Smith).

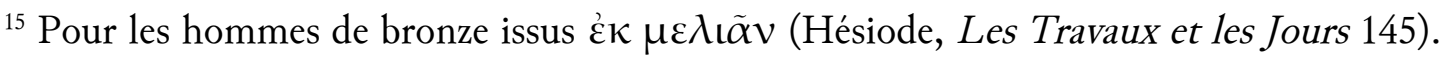

${ }^{16}$ Berndt EFFE, "The similes of Apollonius Rhodius. Intertextuality and Epic Innovation », in Theodore D. Papanghelis, Antonios Rengakos (ed.), A Companion to Apollonius Rhodius, Leyde-Boston-Cologne, 2001, p. 147-169.

${ }^{17}$ Pour la tête, voir Francis Vian, Émile Delage (trad.), Argonautiques (chant III), CUF, Paris, 1980, p. 150 (commentaire à 1398). Pour les pieds, voir Malcom CAmpBell, Studies in the Third Book of Apollonius Rhodius' Argonautica, Hildesheim, 1983 p. 90.

18 Pedro AZARA, "La règle et le serpent ", in La campagne antique : espace sauvage, terre domestiquée, Cahiers Kubaba V, Paris, 2003, p. 53-56. La proximité des représentations entre 
qui s'oppose à la forme visible et ce qui précède la différenciation entre lumière et ténèbres. Qu'un récit de fondation politique comme celui de Thèbes ait recouru à ce genre de représentations n'est pas étonnant : l'autochtonie thébaine devait mettre sur le même plan le monstrueux et l'informe d'un côté, afin de mieux l'opposer à la naissance de l'homme et à la forme de l'autre, ce qui coïncide aussi avec l'émergence de la civilisation. Dans ce cadre, l'anthropogonie et la naissance de la polis sont strictement équivalentes. L'image du serpent venait donc sans doute compléter le principe d'autochtonie en créant un ensemble de représentations des premiers êtres qui était élaboré, complet et cohérent.

Les Présocratiques ne tenaient pas un discours dissemblable. Suivant un principe évolutionniste du temps des origines, ils ont imaginé dans une succession logique l'informe, l'indéfinissable ou le mélangé puis la forme. Eux aussi insistaient sur la différenciation progressive des éléments : nombreuses sont les théories cosmologiques qui attribuaient l'origine de la vie à un mélange d'eau et de terre. Selon Xénophane et Empédocle, les premières créatures seraient nées d'une sorte de fange dont elles se seraient progressivement détachées ${ }^{19}$.

Anaximandre est le Présocratique dont on a gardé une vision relativement claire d'anthropogonie (DK 12 A30) : les hommes seraient nés de l'humidité suivant un processus qui les plaçait d'abord à l'intérieur de sortes de poissons. Ces créatures marines naquirent ellesmêmes de l'eau et d'une terre chaudes et protégèrent dans leur corps les premiers hommes semblables à des embryons, jusqu'à leur arrivé à maturité. Ces derniers furent donc d'abord conçus et nourris dans une substance visqueuse et aqueuse avant de s'en extraire afin de se reproduire comme formes humaines abouties. Cette vision présocratique du cosmos laisse entendre que les hommes se sont dégagés d'éléments naturels comme d'une gaine. L'imaginaire des autochtones accouchés d'une terre maternelle n'est pas très différente.

Ces rapprochements entre deux univers n'ont pas à constituer une fin en soi. L'historien de l'imaginaire qui tente de repérer certains aspects systémiques d'un mode de pensée grec archaïque interroge d'emblée l'écart qui peut exister entre cette cosmologie et certaines croyances orientales. La question a d'autant plus de sens que des influences venues de l'est ont pu intervenir chez les Présocratiques - les Milésiens en particulier. Or, mis à part le rôle générateur de l'eau chez Thalès qui pourrait, à la rigueur, emprunter au Déluge, il demeure par rapport à un certain nombre de cosmologies orientales un écart : l'idée d'un cosmos vivant. Ce principe qui s'observe depuis l'autochtonie thébaine archaïque jusqu'aux théories présocratiques se différencie fondamentalement de l'idée de démiurge. L'émergence des hommes de la terre et/ou de l'eau rejoint d'abord une forme de logique mécaniste de l'apparition de la vie. Il en résulte des lignes de structure cosmologiques en un sens spécifiques, pas forcément représentatives de la totalité des visions grecques des origines; mais celles que nous tentons de cerner au moyen de ces comparaisons composent déjà l'expression cohérente d'une logique de pensée en soi.

Les représentations grecques de phyuê et de phusis précisent ces remarques. Ces conceptions archaïques de croissance et de genèse des créatures s'opposent au schéma de l'apparition des

Python et le serpent d'Arès est d'autant plus forte que l'un et l'autre sont associés à une même divinité, souterraine (ou infernale) et terrible : Telphousa/Tilphossa : voir l'Hymne homérique 276 et la scholie 126 à l'Antigone de Sophocle (Papageorgios).

${ }_{19}$ Voir les fragments DK 21 B33 (Xénophane) et DK 31 A72 (Empédocle) ainsi que Livio Rossetti, Carlo Santaniello (dir.), Studi sul pensiero e sulla lingua di Empedocle, Bari, 2004, p. 55 sq. et p. 403. 
hommes par la volonté des dieux: les mythes de Ptah ou d'Amon dans les cosmogonies memphite et thébaine, ceux de Mardouk et de Nin-Khoursag en Akkad ou de Yahvé en Israël sont caractéristiques à ce titre ${ }^{20}$. L'idée grecque de croissance, quant à elle, renvoie à un fonctionnement cosmique suivant des lois naturelles à strictement parler indépendantes des dieux. Si les Présocratiques ont donné à la phusis un contenu parfois particulier, adapté en outre à leurs principes d'archê, les mythes d'autochtonie déclinaient ce genre de représentations de leur côté, dans une perspective plus traditionnelle. Ainsi, le geste spermique de fécondation de la terre par les dents du serpent d'Arès est, suivant les sources (voir ci-dessus), tantôt accompli par Cadmos tantôt par Athéna. Mais la déesse en elle-même n'est pas considérée comme une divinité démiurge. Un bref détour par l'autochtonie athénienne nous permet d'en être sûr : Érichthonios doit certes sa naissance à Athéna et à Héphaïstos ${ }^{21}$ mais ces derniers n'ont qu'une responsabilité indirecte dans la naissance du premier homme : le sperme du dieu, tombé sur le sol, fait engendrer la terre et c'est d'abord une relation directe entre celle-ci et l'homme qui prime. L'iconographie attique l'a abondamment exposé22.

Autrement dit, ces divinités, contrairement à celles orientales - et judéo-chrétiennes en particulier - s'insèrent dans une vision cosmique plus large où elles n'interviennent pas à titre

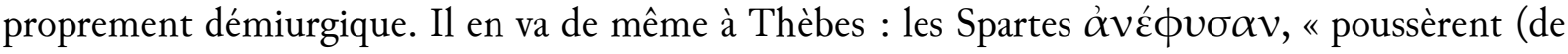
la terre) », exactement comme les arbres suivant un processus en rien différent de ce qu'avançait, par exemple, Empédocle : les premières créatures vivantes furent les arbres, disait-il, qui

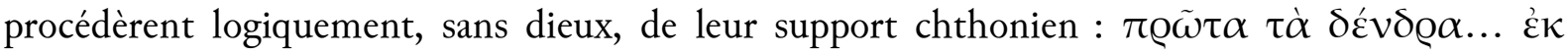
$\gamma \tilde{\eta} \varsigma \alpha \dot{\alpha} \alpha \phi \tilde{v} v \alpha \iota$ (DK 31 A70).

Dans l'un et l'autre cas, l'emploi d'un vocabulaire similaire renvoie à un commun processus créateur. C'est pourquoi les premiers hommes furent perçus, dans l'univers mythologique, comme des sortes de plantes, d'Hésiode - qui chantaient le chalkeion génos « issu des frênes »-

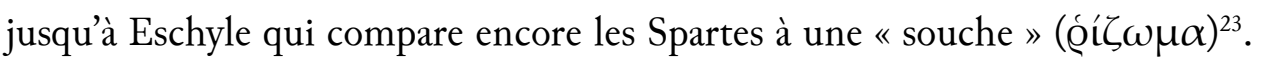

Cette vision de la nature indique finalement comment certaines traditions concevaient la place de l'homme dans le monde : celui-ci a la même relation à la terre que les plantes et les autres créatures. Est-ce un hasard si Aristote, Histoire des animaux I, 6, 491a, classait l'être humain parmi les animaux ? Entre autres représentations grecques, celles que nous étudions ici insistent sur l'idée selon laquelle les êtres sont issus d'un processus physique régulièrement actif, fort éloigné du schéma de la création in abstracto. De ce point de vue l'homme ne semble pas non plus considéré comme une créature bénéficiant par rapport aux autres d'un statut particulier dans le $\operatorname{cosmos}^{24} \ldots$ ce qui ne signifie pas que cette pensée ne soit pas anthropocentrique.

${ }^{20}$ Se reporter à Sources orientales. 1. La naissance du monde, Paris, 1959, p. 66, 68-69, 126, 130, 190.

${ }^{21}$ Voir Euripide, Ion 267-274 ou 999-1003 et les récits synthétiques d'Eustathe, Commentaires à l'lliade II, 283, 25 ou de Hygin, Fables 166.

${ }^{22}$ Pour citer l'ouvrage classique de Nicole Loraux, Les enfants d'Athéna. Idées athéniennes sur la citoyenneté et la division des sexes, Paris, 1981, voir (parmi bien d'autres exemples) les figures 3,4 et 5 a.

${ }^{23}$ Les sept contre Thèbes 413.

${ }^{24}$ Voir Geoffrey E.R. LloYd, Science, Folklore and Ideology. Studies in the Life Sciences in Ancient Greece, Cambridge-London-New York, 1983, p. 26-43. Voir infra sur la spécificité de cette forme d'anthropocentrisme. 
Qu'il fasse l'objet d'un traitement poétique ou d'une expression plus épurée dans le cas d'un certain nombre de présocratiques, cet imaginaire traduit en fait une réflexion sur la forme. $\mathrm{Au}$ principe de genèse - au sens de la création d'êtres vivants par une divinité, suivant les schémas monothéistes dont nous avons hérités - il faut préférer le principe de morphogenèse: l'apparition progressive et logique des formes de vie. La physis intègre implicitement cette conception: elle renvoie à la croissance (d'une plante, d'un être) vue dans sa dimension physiologique : ce n'est pas uniquement la forme observée pour ce qu'elle est, vue de l'extérieur qui importe, mais également la forme comme elle vint à être, comme elle s'épanouit ${ }^{25}$. Placée dans cette perspective de rationalité archaïque, l'autochtonie ne constitue qu'une forme de représentation sophistiquée de plus.

De cet imaginaire que les historiens analysent si souvent à un niveau politique, il ne faut donc pas omettre cet autre aspect : sa dimension cosmologique, existentielle qui s'est sans doute façonnée en harmonie avec le développement d'une vision spécifique du cosmos et de la nature. À cela s'ajoute le fait que cette syntaxe grecque du langage imaginaire ne surexpose pas l'apparition de l'être humain dans l'univers contrairement à l'image d'un Adam dans la Genèse.

Mais on n'a pas tout dit. Si cette anthropogonie s'est développée suivant une vision spécifique du monde, elle a également suivi les courbes d'évolution de l'histoire politique. Mais si l'exploitation politique de l'autochtonie-anthropogonie n'est plus un secret, il faut, en revanche, revenir sur une autre dimension de la cité, évaluer les conséquences que cette cosmologie fait peser sur l'image de la polis. En dehors de l'institution de la patrie, la cité ne gagne-t-elle pas un rôle également cosmologique?

C’est la seconde question à laquelle cette étude propose un début de réponse.

\section{Origines humaines et discours politique : une relation à repenser.}

L'historien des religions qui, occasionnellement, évalue l'autochtonie grecque par rapport à certains récits orientaux constate que c'est finalement "la cité qui fait l'homme" en Grèce. Via le support chthonien qui lui est étroitement associé, la polis ne remplace-t-elle pas de quelque manière le démiurge ? Par ailleurs, il faut considérer que la cité est un socle de constitution historique fondamental de la culture grecque archaïque. Il est généralement admis que la polis prend forme entre les $\mathrm{VIII}^{\mathrm{e}}$ et $\mathrm{VI}^{\mathrm{e}} \mathrm{s}$. av. J.-C : en impactant la pensée grecque, elle pèse donc sur l'univers mythologique comme celui présocratique. Cela ne lui confère-t-elle pas un rôle à la fois sur les plans intellectuel et cosmologique?

Aborder du point de vue grec la cité comme idée, comme entité vue à l'extérieur d'elle-même et sur un plan abstrait, n'est pas si aisé car il ne faudrait pas confondre l'idée que les Grecs classiques en avaient - tout particulièrement les philosophes à compter de Platon et d'Aristote et celle que leurs prédécesseurs archaïques pouvaient en avoir. Pourtant, à y réfléchir, il est possible de renverser le sens de lecture habituellement appliqué à l'autochtonie, en réorientant les relations entre l'autochtonie et la cité du plan politique/idéologique vers un autre, d'ordre

\footnotetext{
${ }^{25}$ Lambros Couloubaritsis, Aux origines de la philosophie européenne. De la pensée archaïque au néoplatonisme, Bruxelles, 200033, p. 59 sq. ou Jean Bollack, Empédocle. Introduction à l'ancienne physique, Paris, 1965, 1, p. 73 sq. Je remercie Arnaud Macé pour ses indications sur une question discutée, reprise par Gérard NADDAF, Le concept de nature chez les présocratiques, Paris, $2008^{2}$ ou Arnaud MACÉ, "La naissance de la nature en Grèce ancienne », in Stéphane Haber, Arnaud Macé (éd.), Anciens et Modernes par-delà nature et société, Besançon, 2012, p. 47-84.
} 
ontologique. La cité elle-même, comme État et comme terre à défendre, masque le propre discours anthropogonique qu'elle génère en occultant sa dimension existentielle derrière des préoccupations sociales et politiques : ainsi l'homme, la créature se trouvent galvaudés au profit du citoyen, du Thébain ou de l'Athénien. Le microcosme, le particulier, cache le macrocosme, la dimension cosmique.

Mais à rappeler que la polis ne fait qu'accompagner le développement d'une vision du monde et de la nature, c'est supposer qu'elle y gagne un statut cosmologique : terreau - dans tous les sens du terme - qui façonne les représentations de l'être humain, la cité semble gagner un rôle d'intermédiaire cosmique entre l'homme et l'univers ; et les autochtones acquièrent en même temps que leur statut politique un statut ontologique.

Comment mettre en valeur ces hypothèses?

Une première façon de souligner le rôle d'intermédiaire cosmique de la cité est de rappeler qu'elle agit comme une grille de lecture du monde.

Il n'y a pas à revenir sur l'autochtonie : la naissance des Spartes coïncide avec la naissance de la cité et de l'identité thébaine, vraisemblablement à travers une association des autochtones à la royauté et à une forme archaïque de citoyenneté : ancêtres des rois mythiques de Thèbes, les Spartes passaient aussi comme ceux de l'ensemble de la communauté politique ${ }^{26}$. Naissance de l'humanité et naissance de la citoyenneté ne sont qu'une seule et même chose. En même temps qu'elle localise l'homme dans l'univers, la cité localise l'homme dans un terroir, une chôra dirions-nous, résultat de la compréhension de la terre sous deux angles différents et complémentaires : la terre-mère qui enfante - des créatures humaines - et, occasionnellement, la terre-père dont il faut défendre les sanctuaires et les bornes - et les Spartes deviennent des prototypes de guerriers ${ }^{27}$.

Mais si les idées de genre sexué habitent les représentations d'autochtonie, il en est d'autres aussi qui agissent : au-delà d'être des Thébains, les Spartes sont en même temps des créatures apparues dans un cosmos incarné par la cité. Cette dernière ne fait que se substituer au macrocosme.

La façon dont la cité opère cette substitution et conditionne une lecture du monde, une sorte de grille de traduction du cosmos se voit encore chez les Présocratiques. Deux exemples sont bien connus : chez Héraclite et chez Anaximandre de Milet.

Dans la pensée d'Héraclite, l'image du monde est calquée sur celle de la cité. Le sage d'Éphèse définissait le cosmos dans le miroir de la polis. DK 22 B94 rapporte par exemple que « le soleil

${ }^{26}$ Une ancestralité restreinte (royale) et large (thébaine) apparait dans les scholies 948 et 949 aux Phéniciennes d'Euripide (Valckenaer) ainsi que dans Eschyle (fr. 731a/b Mette). Ces sources font apparaître le caractère aristocratique de la citoyenneté thébaine archaïque, la « cité » ou le « citoyen » étant vraisemblablement constitué tout d'abord par une élite sociale dont les Spartes sont l'incarnation mythique : sur le régime politique archä̈que de Thèbes, voir par exemple Albert SchachTER, "Kadmos and the implications of the tradition for Boiotian history ", in Gilbert Argoud, Paul Roesch (éd.), La Béotie antique. Colloque de Lyon-Saint-Etienne, mai 1983, Paris, 1985, p. 145-152. Sur la constitution des cités archaïques "à partir du haut", voir Lin Foxhall, "A View from the Top: Evaluating the Solonian Property Classes », in Lynette Mitchell, P. J. Rhodes (ed.), The Development of the Polis in Archaic Greece, London, 1997, p. 113-136.

${ }^{27}$ Sur la dimension paternelle de la terre, voir SeBillotte Cuchet (supra, n. 1), chapitre IX. Sur les Spartes nés tout armés de la terre, voir par exemple Phérécyde, FGrHist 3 F22c. 
ne peut pas dépasser les limites qui lui sont imparties sinon les Érinyes, auxiliaires (غ̇тíkov@oı) de Dikè, le retrouveront ${ }^{28}$. Cette cosmologie, directement inspirée par un fonctionnement civique, ou judiciaire, trouve son point d'orgue dans DK 22 B114 qui use de nomos et de polis :

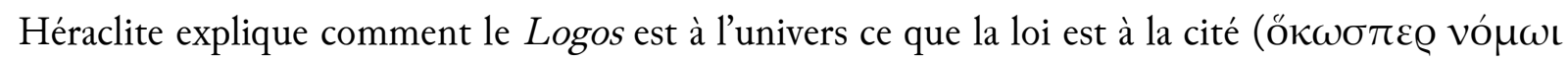
$\tau o ́ \lambda ı \varsigma)$ : régulateur de type législatif agissant au niveau du macrocosme, le Logos, principe spécifique à Héraclite, est ce qui est commun à l'univers et à l'homme... sous-entendu à la cité : celle-ci est interchangeable avec le cosmos et, comme lui, habitacle naturel de l'homme qui s'y intègre tantôt comme citoyen tantôt comme créature. Le Logos, comme la loi, inspire l'homme et le nourrit ${ }^{29}$.

La théorie des saisons d'Anaximandre est un autre exemple illustrant l'antériorité de principe du cosmos sur la polis alors qu'est affirmé, en fait, le modèle de la seconde pour décrire le premier. DK 12 B1 rappelle que le macrocosme est structuré selon des oppositions (le chaud contre le froid) de telle sorte qu'une compensation de l'un contre l'autre forme un tout équilibré. Ainsi s'explique pourquoi certains éléments de la nature disparaissent au profit d'autres. Or, ce processus de rééquilibrage de l'univers est formulé dans les termes d'une responsabilité pénale : si certains éléments disparaissent, c'est parce que d'autres en sont fautifs : ces derniers doivent

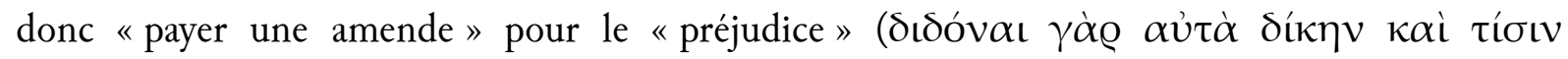

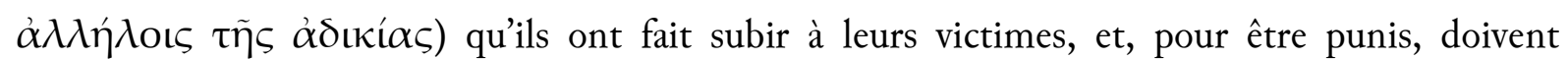
disparaître à leur tour. C'est avec ces termes qu'Anaximandre structurait sa vision du monde ${ }^{30}$ : deux éléments en confrontation constante, le chaud et le froid, disparaissent tour à tour pour expliquer les saisons suivant un processus qui fait intervenir la dikê. Celle-ci régule le cosmos : familiarisé à l'application sociale de la justice, Anaximandre a donc placé la justice dans le domaine cosmique ${ }^{31}$.

Cette manipulation de l'idée de dikê montre que les réflexions d'ordre politique et cosmologique constituent les deux volets d'une même forme de pensée ${ }^{32}$. La vision du monde et le

\begin{abstract}
${ }^{28}$ Sur ce fragment et l'harmoniê selon Héraclite, voir Edward Hussey, « Heraclitus ", in AlgRALONG (supra n. 5), p. 88-112.

${ }^{29}$ Andrea W. Nightingale, "The Philosophers in Archaic Greek Culture ", in H. Alan Shapiro (ed.), The Cambridge Companion to Archaic Greece, Cambridge, 2007, p. 169-198.

${ }^{30}$ Sur l'authenticité de ce fragment : Gabriele GIANNANTONI, «Il concetto di tempo nel mondo antico fino a Platone ", in Giovanni Casertano (dir.), Il concetto di tempo. Atti del XXXII Congresso Nazionale della Società Filosofica Italiana (Caserta, 28 aprile-1 maggio 1995), Naples, 1995, p. 9-23.

${ }^{31}$ Sur l'absence de limites claires entre les réflexions d'ordre philosophique et d'ordre politique : Christopher Gill, Greek Thought, Oxford, 1995, p. 53 sq ; Jula Kerschensteiner, Kosmos. Quellenkritische Untersuchungen zu den Vorsokratikern, Münich, 1962, chapitre I ; Jacqueline DE Romilly, La loi dans la pensée grecque, des origines à Aristote, Paris, 1971, chapitres I et II ; Shirley Darcus Sullivan, Psychological and Ethical Ideas. What Early Greeks say, London-New York-Köln, 1995, chapitre V. Sur la difficulté d'Anaximandre à avancer de nouveaux concepts : Rosemary Wright, Cosmology in Antiquity, London-New York, 1995, p. $61 \mathrm{sq}$.

32 Déjà entrevus par Jean-Pierre Vernant, Les origines de la pensée grecque, Paris, 1962, chapitre VIII, vision nuancée depuis par Ingo W. RATH, "Die griechische Polis als Bezugsrahmen des vorsokratischen Denkens. Ein Versuch der sozial-historischen Verankerung », QUCC 42(3), 1992, p. 63-81 et actualisée par Tobias REICHARDT, "The
\end{abstract}


fonctionnement de l'univers sont conditionnés par des concepts politiques, miroir de la sensibilité des Présocratiques vis-à-vis de l'équilibre et de l'harmonie. Cela donne la mesure de l'influence de la polis sur un autre volet de la vision de l'univers : celui qui s'intéresse à l'homme, à ses origines et à sa place dans ce grand champ cosmique où l'imaginaire cherchait à le situer.

Une seconde façon de souligner le rôle d'intermédiaire cosmique de la cité est de montrer, alors, comment elle constitue la base de réflexion sur la définition de la créature humaine.

À l'époque archaïque, la cité grecque était loin d'atteindre la maturité institutionnelle qui lui est connue pour la période classique ${ }^{33}$. L'observation n'en est que plus intéressante : la polis, d'abord considérée comme une agglomération d'hommes amenés à vivre ensemble - suivant une harmonie sociale décrite en termes de justice ${ }^{34}$-, la polis, donc, existe davantage comme principe organisationnel que comme institution politique. Dans l'idée de cité s'exprime d'abord un type de pensée juridique préoccupé d'équilibre, de dikê ou de moira ${ }^{35}$. Anaximandre ne fit rien d'autre que reprendre certains de ces paramètres régulateurs visant à créer, au plan intellectuel, un tout cosmique harmonieux.

C'est dans ce contexte historique et culturel archaïque qu'intervient également l'autochtonie : l'imaginaire des premiers hommes ne pouvait, lui non plus, se dissocier de ces préoccupations. Le récit des Spartes nous montre le plus précisément combien l'idée (valorisée) d'équilibre et celle (négative) de déséquilibre concerne également les autochtones. Les Thébains archaïques ne pouvaient concevoir de récits originels sans recours à un modèle politique préalable dans lequel leur condition de politês - parcelle d'un tout social organisé - façonnait leur imaginaire et leur condition d'anthrôpos - parcelle d'un tout cosmique.

Des hommes nés de la terre à Thèbes on retiendra en effet deux points.

En premier lieu, une violence excessive les caractérise. Les Spartes sont ces guerriers terribles qui, à peine nés, se combattent et se massacrent mutuellement parce qu'ils sont les descendants du serpent d'Arès. Proches de la race de bronze (Travaux et Jours 143-155) qu'Hésiode décrit comme habitée par l'hubris, les autochtones sont, comme eux, marqués par l'excès et le déséquilibre ${ }^{36}$. Or, Hésiode dit du chalkeion génos qu'il n'est pas durable, pas viable. Les Spartes non plus : vivant par et pour la violence, ils correspondent dans l'imaginaire archaïque à une sorte de créatures originelles prédéfinies qui ignorent le droit ou pour lesquelles la violence tient

Political Institutions of the Archaic and Classical City State as a Breeding Ground of Philosophy », in Marie-Josèphe Werlings, Fabian Schultz (dir.), Débats antiques, Paris, 2011, p. 113-125.

${ }^{33}$ Voir les mises au point du Copenhague Center on the studies on Greek Polis, par exemple Mogens Herman Hansen, "A Survey of the Use of the Word Polis in Archaic and Classical Sources ", in Pernille Flensted-Jensen (ed.), Further Studies in the ancient greek polis, Stuttgart, 2000, p. 173-216.

${ }^{34}$ Se référer à Hésiode, Travaux et Jours 225-247 à propos de la ville florissante, éprise de justice, et de la polis calamiteuse qui dépérit sous l'injustice.

${ }^{35}$ Sur la moira comprise comme la part laissée à chacun, soit une forme de justice, voir Arthur W.H. AdKINs, "Cosmogony and Order in Ancient Greece », in Robin B. Lovin, Franck E. Reynolds (éd.), Cosmogony and Ethical Order. New Studies in Comparative Ethics, Chicago-London, 1985, p. 39-66.

${ }^{36}$ Jean-Pierre Vernant, Mythe et pensée chez les Grecs, Paris, 1985, p. 19-47 récemment commenté par KAPLANIAN (supra n. 2), chapitre VII. 
lieu de droit. Retenons cette forme de réflexion sur la viabilité possible ou impossible des créatures.

En second lieu, ces autochtones ignorent une autre loi étroitement associée au Zeus de la Théogonie : les saisons. On sait comment le Cronide institua les Heures (vers 901-903), créant un écoulement ordonné et harmonieux du temps dans le cosmos. Or, les Spartes, au-delà de leur ignorance de la loi politique de Zeus (= la violence qui tient lieu de justice), ignorent en même temps sa loi cosmique. La croissance des Spartes depuis le sol est en effet excessive : elle s'apparente à celle des plantes exubérantes à la pousse monstrueuse et incontrôlée. Les hommesplantes correspondent à un développement végétal qui ignore totalement le temps cosmique de Zeus. Adultes à peine nés du sol, ils expriment dans leur soudain mode de croissance une continuation éphémère du chaos temporel originel.

Dans ces figures se trouvent d'antiques représentations à la fois politiques et cosmologiques. L'image de la plante exubérante se retrouve dans la pensée de Solon : dans le fragment 13 (West), le législateur archaïque use d'une métaphore chargée de sens lorsqu'il définit l'hubris, l'orgueil humain : elle est comme la pousse précipitée d'une plante qui ne connait pas la limite, ignore la satiété ${ }^{37}$. Cet orgueil est source de stasis, de déséquilibre pour la cité. Et cette association d'idée n'a rien de ponctuel : elle se prolonge parmi les réflexions des botanistes hellénistiques par lesquels un petit détour vaut la peine : quand il parle de la croissance excessive

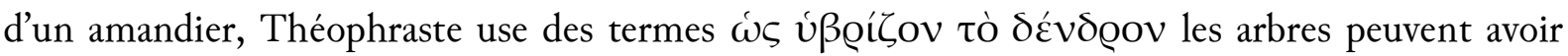
une sorte d'hybris (Causes des plantes II, 7, 6). Lorsque l'élève d'Aristote parle d'une variété de vignes "appelées folles ", il écrit : "elles ne font pas seulement croitre, mais font pousser aussi [...] des fleurs et des grappes sans qu'elles aient le pouvoir de les faire mûrir » $(\mathrm{I}, 18,4)^{38}$. L'absence de mesure, qui est « hybris » ou «folie », mène inévitablement à la maladie comme le soulignait encore Platon (Lois 691c) : «si l'on donne [...] en dépassant la mesure, que ce soit [...] de la nourriture aux corps ou de l'autorité aux âmes, tout sombre, et dans leurs excès

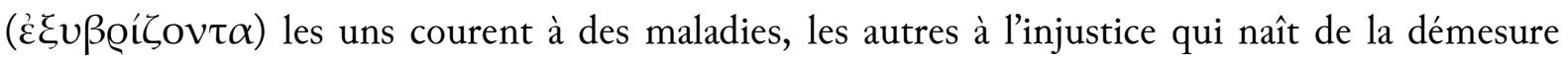

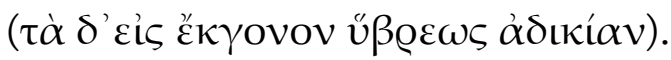

Qu'elles soient de forme humaine ou de forme végétale, les créatures hybristiques sont causées par une trophê excessive : elles ne portent pas de fruits ou ne les mènent pas à maturité. Elles deviennent comme «folles » en croissant au-delà de la normalité et s'autodétruisent : c'est trait pour trait l'image des archaïques Spartes dont la germination démesurée traduit indiscutablement le comportement hybristique : race violente, chaotique et excessive, vouée à sa propre perte et non-humaine... s'il n'était une forme d'équilibre qui en sauva cinq pour en faire les fondateurs de la cité et les ancêtres des Thébains.

Cet imaginaire exprime à sa façon l'importance d'une harmonie à atteindre pour que la cité, et les hommes avec elle, existent. Indépendamment de l'époque à laquelle cette idée politique d'harmonie a pu naître, l'enseignement à retenir est d'abord celui-là : la polis est la seule capable d'intégrer les créatures dans l'humanité - de fabriquer, en somme, les hommes. On notera lesquels parmi ces autochtones sont considérés comme des hommes à part entière : cinq Spartes survivants du massacre, considérés comme seuls capables d'engendrer des descendants humains : ces autochtones-là, et eux seuls, ont surmonté le chaos alors que leurs frères, aussitôt morts dès

\footnotetext{
37 À l'inverse, l'image de l'épanouissement végétal est utilisée pour définir la dikè : voir Jonathan A. AlmeidA, Justice as an Aspect of the Polis Idea in Solon's Political Poems, LeidenBoston, 2003, p. 209 sq.

${ }^{38}$ Sur ce thème, voir Ann MicheLINI, " $\Upsilon B P I \Sigma$ and Plants ", HSPh 82, 1978, p. 35-44.
} 
leur naissance, ont sombré dans la folie et l'excès. En tant que référence idéologique pour les Thébains, ce paradigme semble ambigu mais il s'éclaire dès lors que la cité est considérée comme la référence cosmologique qui orchestre in fine une "fabrication" de l'humanité illustrée par deux groupes différents d'autochtones.

Ceux parmi les Spartes qui s'entretuent, à cause de leur folie, donnent l'image de pré-humains, de presque humains : ils sont des hommes "imparfaits". Employons fortement les guillemets en usant du terme imparfaits car ils s'imposent par défaut, cette “imperfection” n'ayant rien en commun avec la vision judéo-chrétienne nourrie de l'idée de la créature parfaite à l'image de son créateur. Cette forme d'incomplétude a un caractère à la fois juridique et ontologique : les Spartes qui meurent sont "imparfaits" en ce qu'ils sont presque des ancêtres politiques comme ils sont presque des humains. Ils constituent une race fragilisée par l'absence de droit et appelée, pour cette raison même, à disparaitre. Sur ce point, la même logique s'applique aussi pour le chalkeion génos d'Hésiode. Au niveau de lecture anthropogonique, les Spartes qui s'entretuent sont finalement moins des hommes que des monstres, correspondent à une étrange race intermédiaire incapable de générer des descendants humains, inaptes à fonder une cité. Ils sont plus proches du serpent furieux d'Arès, de l'informe, que leurs congénères qui réussissent, eux, une forme de passage et qui intègrent l'humanité puisqu'ils génèrent des enfants. Ces autochtones survivants expriment une forme d'équilibre, correspondent à une harmonie sociale primordiale et sont pleinement humains.

Au niveau explicite le récit opère bien une équation entre naissance de la cité et naissance de l'humanité ; au niveau implicite, il construit un discours cosmologique où le statut humain découle de l'insertion de la créature dans la cité. La polis est un curseur du statut ontologique des créatures. Elle tient le rôle d'un intermédiaire cosmique suivant une logique des représentations archaïques remarquablement cohérente.

On notera combien la réflexion sur l'être humain est guidée par un souci d'équilibre, du type formulé par Anaximandre. On ne saurait bien sûr affirmer que l'imaginaire thébain découle des théories présocratiques mais on soulignera un saisissant parallèle. Dans le cas de Thèbes, l'équilibre - qui est par ailleurs exprimé par la déesse Harmonia - ne peut être atteint qu'au moyen d'une confrontation entre des éléments amenés à disparaitre (les Spartes qui meurent) et d'autres amenés à subsister (les Spartes humains fondateurs de la cité) : ces derniers forment comme une sorte de compensation à l'hubris initiale. Mais au risque d'appliquer une lecture présocratique au mythe, on formulera davantage les idées comme suit : les survivants reflètent une sorte d'équilibre qui permet à la créature humaine de véritablement naitre dans le cosmos. La folie et l'excès passés, les autochtones deviennent des êtres viables. Cette idée de viabilité constitue une réflexion à la fois cosmologique et politique : il y un enjeu anthropogonique - les autochtones deviennent des humains à part entière, des êtres viables, intégrables dans un univers ordonné - et un enjeu communautaire - les autochtones deviennent en même temps des ancêtres et des pères politiques, garants d'un bon ordonnancement du temps et d'une fondation de la cité. La nature des Spartes se paramètre bien en fonction de la cité comme grille de lecture $\mathrm{du}$ monde et en fonction de ses valeurs politiques : la correspondance entre le politês et l'anthrôpos est parfaite. Situer l'homme dans le tout cosmique va de pair avec le fait de le situer dans le tout social. L'homme est d'abord l'élément constitutif d'un tout équilibré qui le fonde comme être humain.

Derrière cette structuration de l'imaginaire se lit l'ordonnancement progressif d'une pensée politique qui régule la société en même temps que l'humanité, en délimite les contours. À mesure que s'impose l'idée de cohésion du groupe social s'affirme aussi celle de l'humanité 
entière : les Spartes fous et monstrueux constituent une forme de scorie que la polis écarte d'entrée de jeu du statut humain : elle ne les admet pas en son sein.

De ce point de vue, la cité apparait donc comme l'intermédiaire entre l'homme et l'univers : elle paramètre une logique qui assure la promotion ou le déclassement de la créature dans la hiérarchie des êtres. La polis, dès l'époque archaïque est, en cela, une référence ontologique et cosmologique.

C'est dire, alors, que les identités politique et ontologique de l'être humain se confondent. La polis constitue en elle-même une image aboutie du cosmos. Ce statut implicite de la cité mis à découvert, nous comprenons alors mieux pourquoi, dans cette logique de pensée, l'homme se confond à une plante qui pousse sur une terre : il phuei, s'épanouit dans son cadre naturel, la "polis-cosmos". Dans cette vision du monde où la cité est le paramètre de définition ontologique de l'humain, la polis remplit le rôle tenu ailleurs par le démiurge.

\section{La cité grecque et l'anthropologue : quelques pistes de réflexion}

La constitution historique de la cité et sa dimension identitaire et idéologique, fondamentale dans la constitution des communautés grecques archaïques, semble avoir déplacé les réflexions sur la créature humaine (en tant que telle) à la lisière des croyances. Cependant, il ne serait possible d'en faire l'absolue affirmation tant la contiguiité des représentations politiques et cosmologiques empêche de plaider pour une franche marginalité des réflexions ontologiques.

De même, le caractère géographiquement dispersé des récits d'autochtonie pourrait faire croire au caractère non-anthropocentrique de la pensée grecque archaïque. Mais là encore, ce n'est qu'apparence, renforcée par la logique mécaniste d'une vision du monde à laquelle l'autochtonie participe. Ces traditions, certes détournées du souci de la création canonique d'un premier homme universel, à l'image d'un Adam, sont le résultat d'une gestation historiquement lente et géographiquement diffuse de l'institution politique. De même, la comparaison du schéma d'autochtonie à quelques unes des croyances anthropogoniques orientales n'engage, à l'intérieur d'une culture grecque bigarrée, que la particularité d'une tradition parmi d'autres où l'homme, sorte d'ersatz végétal, semble n'avoir rien d'exceptionnel au plan cosmologique. Et pourtant, là encore, agit une forme de compensation dans la mesure où l'homme-plante correspond à une forme d'exception en tant qu'homme politique : l'être humain, s'il n'est pas surexposé à l'échelle cosmique, l'est en revanche au plan politique. On se souvient des Athéniens d'époque classique qui tiraient une grande fierté de leur autochtonie au point de s'affirmer comme supérieurs au reste des $\mathrm{Grecs}^{39}$. De manière plus générale, les récits d'autochtonie font intervenir la nécessité pour les hommes de se raccorder à la cité et de s'affirmer par son biais comme des êtres humains, au centre du monde. C'est, par rapport aux représentations judaïques et chrétiennes, une autre structuration de l'anthropocentrisme : l'affirmation de la situation de l'homme au centre du cosmos est bien là mais biaisée (dans tous les sens du terme) puisque la cité se substitue au cosmos et se confond avec lui.

Mettre l'autochtonie en perspective avec l'histoire de la pensée grecque archaïque fait apparaitre la cité comme le prisme culturel et intellectuel au travers duquel le regard porté sur l'univers et sur l'homme s'est construit. Dans ce cas, c'est peu de souligner le rôle structurant de la cité au niveau du développement de représentations cosmologiques: la polis pose un problème philosophique implicite : celui de la viabilité et de l'existence même des hommes.

${ }^{39}$ Voir les paroles de l'Athénien Syagros face à Gélon dans Hérodote, Enquête VII, 161. 
Elle le fait en termes de vécu quotidien, portée par la nécessité de l'harmonie sociale ; et elle le fait en termes ontologiques. La cité fait donc plus que donner à l'homme grec une identité politique : elle lui donne plus largement une place dans le monde et, en même temps, la polis en acquiert une pour elle. Extraite de sa dimension et de son rôle politiques (ordinaires), la cité se dote d'un statut d'ordre spirituel en tant que médiatrice entre l'homme et l'univers : elle est ce par quoi se définit l'informe et la forme, le chaos et le cosmos, le civilisé et le non-civilisé. Cette vision du monde, ce besoin de polis pour réaliser son humanité, se présente encore dans Eschyle, Les Sept contre Thèbes 376-396, 412-414 et 473, où les descendants des Spartes, les citoyens Mégareus et Mélanippe, défendent les portes Proitides devant le furieux et chaotique

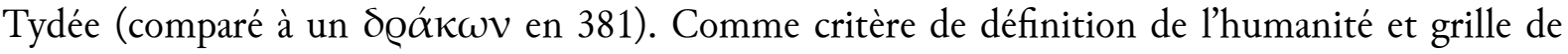
lecture du monde, la cité de Thèbes est ce par quoi s'érige l'humain et le non-humain et, plus largement, le sens.

Ces remarques peuvent être prolongées sur le plan des cultes où le même discours cosmologique et ontologique est présent à un niveau implicite des pratiques, des croyances.

Les fêtes ne manquent pas où un parallèle est établi entre vie humaine et vie végétale comme dans les Hyacinthies de Sparte où d'anciennes traditions associaient le héros à la terre et à la floraison ${ }^{40}$ alors que, en même temps, l'événement avait une signification politique importante du fait de la participation de tous les citoyens ${ }^{41}$. Dans le même ordre d'idées la confection de l'eiresiônê aux Thargélies et Pyanepsies d'Athènes prête matière à réflexion : ce rameau ornés des fuits de la terre appelés hôrait était associé aux paides. Plutarque voyait dans la participation des jeunes à cette fête une réminiscence de Thésée, fondateur de la cité ${ }^{43}$ mais la convergence est tout aussi intéressante entre les paides et les jeunes pousses mises en valeur par les hôrai : n'y a-t-il pas dans ces rites, à la fois agraires et civiques, l'affirmation d'une adhésion des hommes et de la végétation au temps cosmique ? L'adéquation des calendriers agraire et politique ne renverrait-il pas une certaine image de la créature humaine : celle de sa condition dans l'univers qui s'exprime à travers la polis ?

Le serment des éphèbes à Athènes, tel qu'il nous est connu par la stèle d'Acharnes ${ }^{44}$, est sans doute le document qui pose avec le plus d'acuité ces questions. L'intérêt du texte est de laisser, à côté de divinités guerrières, une place importante à d'autres $\theta \varepsilon o$ ó (1.1), parmi lesquels 'A $\gamma \lambda \alpha v 0 o s, \Theta \alpha \lambda \lambda \omega$, A $v \hat{\xi} \omega$, 'H $\Theta \varepsilon \mu o ́ v \eta$ (1. 17-19) dont H. Jeanmaire a expliqué la présence en raison de leur rôle courotrophe ${ }^{45}$. Ces "Charites", nourricières, présentes dans des croyances où affleure une image à la fois maternelle et paternelle de la terre, indiquent, au-delà de la nécessité de défendre le territoire, la relation de foi profonde qui, sans doute, unit l'homme au

\footnotetext{
${ }^{40}$ Voir Pausanias, Périégèse III, 19, 4 (aspects sans doute archaïques de Hyacinthos), de même que l'analyse de Jacqueline Christien, Françoise Ruzé, Sparte. Géographie, mythes et histoire, Paris, 2007, p. 17-18.

${ }^{41}$ Hérodote, Enquête IX, 7, 11.

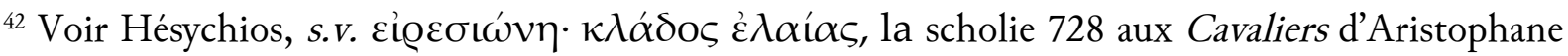
et Montserrat CAmps-Gaset, L'année des Grecs : la fête et le mythe, Paris-Besançon, 1994, p. $77-79$.

${ }^{43}$ Vie de Thésée XXII.

${ }^{44}$ Voir l'édition récente de P. J. Rhodes, Robin Osborne (ed.), Greek Historical Inscriptions 404-323 BC, Oxford, 2003, $\mathrm{n}^{\circ} 88$.

${ }^{45}$ Henri Jeanmaire, Couroi et Courètes, New York, 1975 [Lille, 1939], p. 309.
} 
sol. R. Parker a fait remarquer d'une façon lapidaire comment ces divinités de la végétation, interchangeables avec les Heures qui permettent la croissance des plantes, sont aussi des déesses "of human growth»: les éphèbes les honorent "partly because it is their duty to protect the produce of the land, and partly because they are themselves a portion of that produce ${ }^{46} »$.

Reste à préciser combien le devoir civique des futurs hoplites se comprenait aussi dans une perspective cosmologique : la terre, valeur patriotique, était l'entité cosmique et la mère d'où les hommes étaient nés. Il se peut que la stèle d'Acharnes réifie donc une image de l'être humain issu des principes actifs de l'univers dont les divinités, Charites ou Horai, assuraient le fonctionnement. Investi du devoir de défendre sa terre, le citoyen athénien était susceptible de se rappeler, ainsi, sa condition humaine car il contribuait simultanément au maintien de l'ordre cosmique. Ce genre de rite constituait vraisemblablement une forme de réflexion sur la condition humaine situé à cheval entre représentations politiques et ontologiques, comme les mythes d'autochtonie.

À partir du traitement de l'autochtonie par les Phéniciennes d'Euripide, V. Sebillotte Cuchet a insisté sur l'idée de dette qui s'exprimait dans le cas de Ménécée, descendant des Spartes : la pièce développe à souhait la nécessité d'un don en retour de la vie donnée par la terre ce qui conduit au suicide du jeune homme ${ }^{47}$. Cette donnée explicite en fait une forme de représentation cosmologique valable en Grèce comme dans d'autres religions : celle où la créature humaine est liée au principe de création du monde ou de créateur par une forme de redevance. Ainsi, chez les Babyloniens, l'humanité se définit comme un servage existentiel car elle a été fabriquée pour travailler à la place des dieux. Dans l'optique judéo-chrétienne, la dette, liée au péché originel, se confond au devoir d'obéissance absolu à la volonté divine et à l'acceptation de la faiblesse du libre-arbitre. Autrement plus explicite est la redevance dans l'optique précolombienne : chez les Aztèques cette dette prend la forme de mutilations rituelles en retour du sang versé par les dieux qui créèrent le premier homme ${ }^{48}$. En Grèce, l'usage politique qui est fait de l'autochtonie déplace cette dette vers le devoir civique mais la dimension cosmique n'en semble pas évacuée pour autant : en défendant le territoire, le citoyen s'inscrit aussi dans le cosmos, rappelant par son devoir politique les liens particuliers qui l'unissent à la terre.

Les Spartes, enfin, loin de l'ambiguïté entre prestige autochtone et meurtre - une ambivalence à laquelle la tragédie attique nous a habitués - n'avaient peut-être rien de si contradictoire : ils rappelaient aux citoyens thébains leurs origines ainsi que la nécessité de la guerre qui lie l'homme à la terre et qui explique pourquoi une partie d'entre eux doit retourner dans la matrice originelle. Il est possible que cette vision du monde ait été répandue ailleurs en Grèce, bien audelà des cités se réclamant de l'autochtonie. La gestuelle d'un rite crétois, à Dréros, ne laisse pas de réfléchir: les éphèbes plantaient un olivier au terme d'une initiation guerrière et d'un serment : qualifié d'« obscure et d'archaïque » par R.F. Willetts ${ }^{49}$, cette pratique ne rappelait-elle

\footnotetext{
${ }^{46}$ Robert Parker, Polytheism and Society at Athens, Oxford, 2005, p. 435.

47 Violaine Sebillotte-Cuchet, «La terre-mère : une lecture par le genre et la rhétorique patriotique », Kernos 18, 2005, p. 203-218 (en particulier p. 215).

48 Sur le «nextlanalitztli », traduit comme «sacrifice de sang », voir Michel GraULich, « Les Aztèques avaient-ils une religion ? ", in Ugo Bianchi (ed.), The Notion of "Religion» in comparative Research. Selected Proceedings of the XVI International Association for the History of Religions Congress. Rome, $3^{\text {rd }}-8^{\text {th }}$ September, 1990, Roma, 1994, p. 239-245.

${ }^{49}$ Cretan Cults and Festivals, London, 1962, p. 201. Pour une critique de Willetts, voir Marcel Detienne, «L'olivier : un mythe politico-religieux », in Moses I. Finley (dir.), Problèmes de la
} 
pas à l'éphèbe sa double identité : celle d'un nouveau politès lié à sa terre comme le nouvel arbre ? Et aussi celle d'un anthrôpos plongeant dans la terre les mêmes racines que le végétal ? Dans cette forme de pensée, définir la nature profonde de l'homme c'est bien définir sa place dans l'environnement immédiat. Comme anthropogonie bien à part, l'autochtonie est le produit d'une histoire investie de rationalité, de croyances et de schémas cosmologiques bien éloignés de l'idée judéo-chrétienne du créateur. En lieu et place se trouve un comportement, une culture politique où s'épanouit le sens de la morale, de la spiritualité, de l'imaginaire, de l'identité, le sens du monde. Il n'y a rien dans cette forme de pensée qui motive, comme nous le concevrions dans notre culture héritière du judéo-christianisme, un Adam originel ; mais l'imaginaire d'anthropogonie est bien là, donnant autrement à l'homme toute la mesure de son caractère unique lequel est caché derrière la patrios politeia et les idées sur la citoyenneté et le sexe.

terre en Grèce ancienne, Paris-La Haye, 1973, p. 293-306. 